Revue trimestrielle sur l'image géographique et les formes du territoire

\title{
La géographie, ça sert aussi à écrire des romans : l'impact des choix géographiques sur la trame narrative dans la saga Game of Thrones
}

\section{Philippe Moyen}

\section{(2) OpenEdition}

\section{Journals}

Édition électronique

URL : http://journals.openedition.org/mappemonde/3692

DOI : 10.4000/mappemonde.3692

ISSN : 1769-7298

Éditeur

UMR ESPACE

Référence électronique

Philippe Moyen, «La géographie, ça sert aussi à écrire des romans : l'impact des choix géographiques sur la trame narrative dans la saga Game of Thrones », Mappemonde [En ligne], 121 | 2017, mis en ligne le 01 juillet 2017, consulté le 21 décembre 2020. URL : http://journals.openedition.org/mappemonde/ 3692 ; DOI : https://doi.org/10.4000/mappemonde.3692

Ce document a été généré automatiquement le 21 décembre 2020.

\section{(i) (2)}

La revue Mappemonde est mise à disposition selon les termes de la Licence Creative Commons Attribution - Pas d'Utilisation Commerciale - Partage dans les Mêmes Conditions 4.0 International. 


\title{
La géographie, ça sert aussi à écrire des romans : l'impact des choix géographiques sur la trame narrative dans la saga Game of Thrones
}

\author{
Philippe Moyen
}

"La géographie sert d'abord à faire la guerre ", cet aphorisme bien connu d'Yves Lacoste a été repris de manière inattendue par de nombreux auteurs de fictions d'Heroic Fantasy. Ce genre littéraire, longtemps méprisé au sein de la communauté universitaire, semble accéder aujourd'hui à une reconnaissance tardive en particulier grâce aux succès cinématographiques et télévisuels des œuvres de Tolkien et Martin. Les spécialistes de littérature et d'histoire glosent sur les richesses insoupçonnées de la trame narrative et des inférences historiques de ces récits (Rolet, 2014). Si les géographes ont déjà étudié les liens entre les territoires, leurs représentations et les fictions littéraires (Géographie et littérature, http://cafe-geo.net/geographie-etlitterature-2), leur silence peut surprendre alors que les auteurs d'Heroic Fantasy ont le souci d'inscrire leurs intrigues dans des mondes textuels complexes avec leurs lieux et leurs pratiques spatiales. Fort de ces remarques liminales, il semble légitime de questionner les partis pris de Martin à l'œuvre dans la constitution du monde de Game of Thrones de manière à mettre en évidence les interactions entre l'imaginaire géographique de l'auteur et une culture géographique, largement héritée du cursus scolaire et de travaux plus savants, qui nourrissent le récit et pèsent sur les horizons de réception de l'histoire auprès du public. Pour traiter cette problématique, j'ai opté pour une démarche classique articulée autour de trois grands axes thématiques: le cadre naturel, les territoires et les pratiques spatiales. Le choix de Game of Thrones est commode, il surfe sur le formidable engouement provoqué par la mise à l'écran par la chaîne HBO de cette saga et constitue, à ce titre, un outil pratique pour bâtir une herméneutique de la géographie d'une œuvre de fiction littéraire. Conscient de céder à 
une forme de réductionnisme, je me réfèrerai, dans cet article, plutôt à l'adaptation télévisée qu'aux romans, à l'exception de la carte du monde proposée par l'auteur dans ses livres et absente à l'écran. Ce choix se justifie par l'énorme audience de la série, en moyenne 23 millions de téléspectateurs par épisode pour la saison 6 et par les quelques 3000 heures de diffusion cumulées depuis le lancement de la série. Le support télévisuel constitue donc un vecteur plus important que les romans des choix géographiques de Martin auprès de son public.

\section{Le cadre géographique du monde de Game of Thrones}

2 Si le générique de la série présente aux téléspectateurs un montage virtuel d'un territoire de jeu de plateau étudié par Bertrand Pleven (in Robic M.-C., Rosemberg, 2016), G.R.R. Martin propose en revanche à ses lecteurs une carte de son monde qui en dit beaucoup plus sur sa manière d'appréhender l'arrière-plan géographique du cadre des intrigues développées. Point n'est besoin de s'étendre sur les péripéties de Game of Thrones, elles sont bien connues du public concerné et, pour les néophytes, la consultation des nombreux sites consacrés à la série sufFIra pour éprouver l'analyse conduite ici.

\section{La carte du monde de Martin}

Le monde de Game of Thrones est représenté sur une carte, une mappemonde de facture classique, mais incomplète et lacunaire. La projection est centrée délibérément sur le « détroit » qui sépare deux grands ensembles continentaux : Westeros et Essos. Ce choix correspond à la volonté de l'auteur de faire coïncider la densité événementielle avec le barycentre du monde tel qu'il veut le représenter. Une analyse plus fine des caractéristiques des différents types d'établissements humains permettra de mettre en évidence un modèle des dynamiques géohistoriques porteur de fortes potentialités aventurières. Sur la carte le Nord est placé en haut, cette convention géographique héritée d'Ératosthène, qui peut sembler évidente, est pourtant signifiante. L'absence des grands repères géographiques que sont latitude et longitude n'occulte cependant pas une certaine zonation bioclimatique. Le Nord est le monde du froid, toujours placé sous la menace d'un hiver tenace. La terre y est ingrate et la vie rude, alors que vers le Sud les aménités d'un climat plus chaud permettent des cultures variées et abondantes. Ce gradient climatique nord-sud correspond aussi à une échelle de moralité des populations qui fréquentent ces lieux. À la droiture virile et austère des populations «nordiennes » s'opposent le raffinement, l'hypocrisie et le relâchement des mœurs des populations du Sud, autant de comportements implicitement liés à la moiteur du climat, la luxuriance des jardins. Dirficile dès lors de ne pas faire le rapprochement entre ce déterminisme du milieu et la théorie des climats chère à Montesquieu et avant lui à Aristote ou Ibn Khaldoun. Ainsi, il existerait chez Martin une volonté de présenter le nord de Westeros comme le conservatoire des qualités humaines jadis communes aux Hommes et aujourd'hui corrompues dans les contrées plus méridionales. Dans la saga, la dynastie Stark incarne les cœurs purs et vaillants du royaume des sept couronnes à l'opposé des Lannister présentés comme fourbes, incestueux et cupides. 


\section{Le continent de Westeros}

4 Le monde de Martin est constitué pour l'essentiel de deux grands ensembles d'échelle continentale et d'archipels situés dans les mers bordurières. Westeros est le continent le plus occidental. Il s'agit d'un vaste espace étiré du nord au sud sur une superficie voisine de l'Amérique latine même si sa morphologie évoque davantage la GrandeBretagne. L'étirement des distances, compte tenu des moyens de transport disponibles (chevaux, chariots), et de l'état des routes, permet à l'auteur de dégager les durées nécessaires à la mise en œuvre des séries de rebondissements qui alimentent la trame de son récit : trahisons, renversements d'alliances, reconstitutions des forces... Chaque voyage devient un périple voire une quête, l'espace parcouru permet ainsi une évolution des personnages, ce qui renouvelle le récit. Les pérégrinations de Jaime Lannister et de Brienne de Torne sont à cet égard tout à fait exemplaires. La fidèle et dévouée Brienne a pour mission d'accompagner le fils aîné Lannister à Port Réal pour l'échanger contre les filles Starck retenues en otages dans la capitale. Jaime Lannister est dépeint comme cynique, cruel et fourbe. Au fil de leur voyage, la nécessité de faire face aux périls rapproche les deux personnages et une complicité inattendue se tisse entre eux. Seule la durée du voyage, liée à l'étirement des distances, permet à l'auteur d'opérer cette mutation de la psychologie de Jaime Lannister pour mieux relancer l'intérêt du public pour un personnage trop vite confiné au simple rôle d'assassin du dernier roi Targaryen.

\section{Le continent Essos}

5 L'autre continent, Essos, est un bloc compact étiré d'ouest en est dont la morphologie et les caractéristiques bioclimatiques se rapprochent de l'Asie. Le cœur continental est constitué d'une vaste plaine appelée «mer herbeuse » encadrée au nord par une région glacée et au sud par le " désert rouge » et la « mer d'été ». Les littoraux méridionaux et occidentaux sont découpés en profondes baies susceptibles d'accueillir des installations portuaires conséquentes. Dans le monde de Martin, cet orient est nimbé d'exotisme et de mystères. Violence, magie et rafFInement s'y imbriquent sans distinction au sein de sociétés esclavagistes dont les valeurs sont jugées avec condescendance par les rares Westriens qui osent s'aventurer dans ce «Far East ». Cet espace ne se distingue guère des topiques littéraires de Marco Polo, Montesquieu ou des auteurs romantiques du XIX siècle. Il n'est donc pas surprenant d'y retrouver les archétypes du Khan mongol (Khal Drogo), à la tête de sa horde, des mages (les Non-Mourants de la cité de Qarth), des sectes d'assassins (les Navrés/les spadassins de la Demeure du Noir et du Blanc de Braavos) ou des guildes de marchands peccamineux (les Judicieux de la cité de Yunkaï).

\section{Le Mur et le Détroit}

6 S'il semble malaisé de distinguer des frontières sur la carte, en revanche, la partition du monde de Martin relève d'une conception très classique des frontières fondée sur des discontinuités de géographie physique. À cet égard deux lieux doivent retenir l'attention. D'abord, l'isthme septentrional de Westeros, entre la « mer du Crépuscule » et la "mer Grelotte », barré par un gigantesque mur de glace édifié dans un passé immémorial, sépare le monde connu des contrées inconnues. Puis l'outre mur, une terre glacée en permanence, une limite de l'œkoumène, peuplée de sauvages et de 
créatures légendaires (les marcheurs blancs ou l'armée des morts qui ne peut qu'évoquer les einherjar des mythologies nordiques). Comme souvent ces marges contiennent un potentiel dramatique important à l'exemple de la forêt de Brocéliande ou du désert des Tartares. L'histoire y change d'échelle pour devenir une véritable guerre des mondes dont l'enjeu n'est rien de moins que la survie de l'Humanité. Dans le monde textuel de Martin, le Mur est une frontière topographique, mais aussi politique entre le monde ordonné du Royaume des Sept Couronnes et les territoires des Peuples Libres tenus par des chefFeries turbulentes et rivales. L'autre grande dissymétrie est le détroit de Westeros. Si cette discontinuité apparait d'emblée moins absolue que la précédente, elle n'en demeure pas moins importante pour le récit. Le détroit sépare deux rivages bien distincts en termes de densité de population, d'organisation du territoire et de pratiques spatiales.

Figure 1. Schéma du monde de Game of Thrones

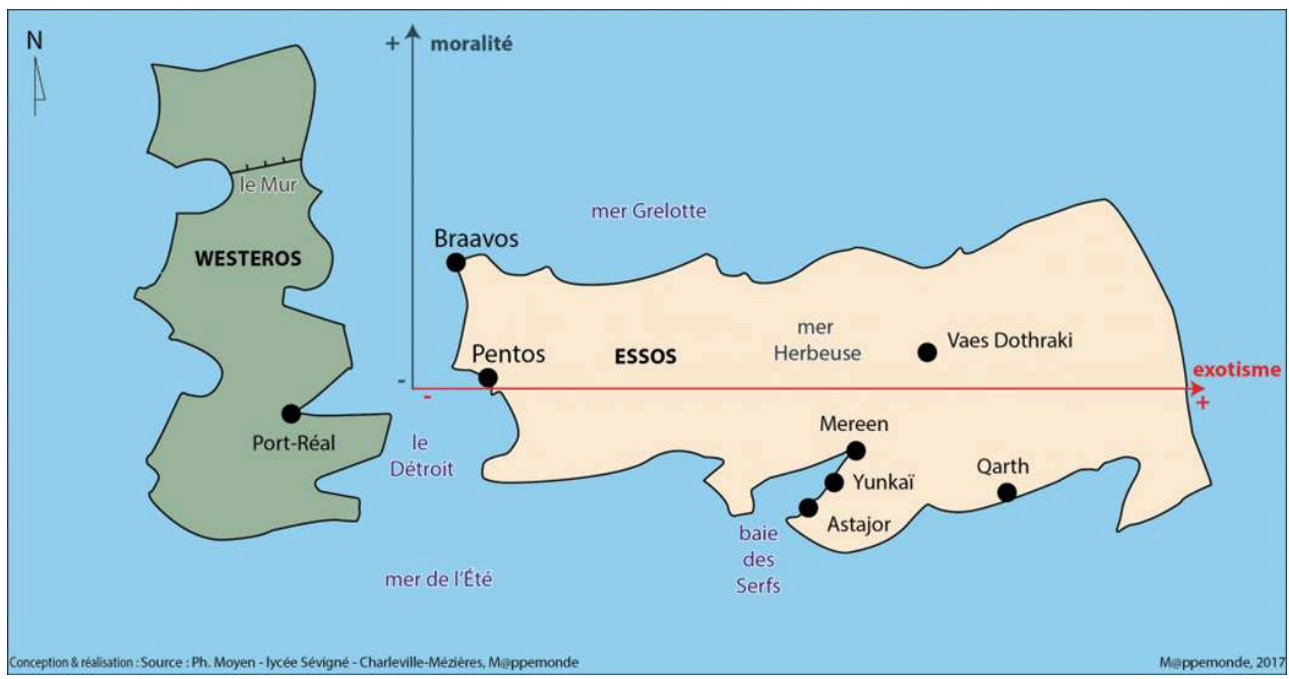

\section{La diversité des territoires}

La notion de territoire renvoie à la définition classique proposée par Roger Brunet, un espace approprié (Brunet et al., 1993). Pour l'essentiel, il s'agit d'un ensemble d'actions d'abrègement, d'afFectation et d'identification exercées sur un espace par une société. L'importance de l'arrière-plan politique et géopolitique de la saga Martin invite à une recension des différents types d'établissements humains installés à Westeros et à Essos pour pouvoir mettre en évidence des dynamiques géopolitiques susceptibles de renforcer la densité événementielle dans le récit. Quatre grands types d'établissements humains peuvent être distingués dans le monde de Martin à partir de leurs structures politiques : un royaume centralisé, un vaste empire nomade, des cités libres et enfin des chefFeries archaïques. Les choix géographiques de l'auteur de la saga ont largement déterminé l'implantation et la morphologie des établissements humains recensés.

\section{Port Réal et les terres de la couronne}

La plus grande partie de Westeros est occupée par les sept royaumes unis en un État centralisé et gouverné depuis la capitale de Port Réal par les successeurs Lannister de 
feu le roi Robert Barathéon. La sédentarisation du pouvoir incite à comparer le royaume du Trône de Fer à une monarchie européenne de l'époque moderne, du type de l'Espagne de Philippe II ou de la France de Louis XIV, capable de gouverner au loin grâce à une administration très développée, disposant de nombreux relais en province pour veiller à l'exécution de la volonté du souverain. La ville de Port Réal est bâtie sur la rive nord du delta de la Néra. Ceint d'une muraille percée de sept portes, l'espace intra-muros propose une division sociospatiale verticale du territoire. Au sommet des trois collines sont juchés le Grand Septuaire, le palais royal et le Donjon Rouge, siège du conseil. Au pied des collines, la ville offre un autre visage : la fourmilière crasseuse de Culpucier. Lorsque le Tribunal de la Foi condamne la reine Cersei Lannister à expier ses fautes, celle-ci doit parcourir nue et tondue les rues de Port Réal sous les quolibets d'une foule haineuse en une procession symbolique d'humiliation et d'abaissement social pour rejoindre son palais, purifiée de ses erreurs passées.

\section{Le monde féodal du Nord et « l'Outre-Mur »}

Lorsque l'intrigue se déplace de la capitale vers le Nord, le lecteur se retrouve plongé dans un monde médiéval de châteaux forts contrôlés par des maisons seigneuriales d'importance variable et liées au pouvoir central par des relations féodo-vassaliques. Cette organisation du territoire est le fruit d'un abrègement territorial ancien destiné à préparer une défense régionale face à la menace de l'Outre-Mur qui hypothèque l'avenir de l'Humanité lorsque le long hiver redouté viendra. Dans cet espace féodal, les mariages revêtent une importance capitale dans les stratégies territoriales de ces seigneurs. Cette distorsion spatio-temporelle entre les difFérentes structures étatiques de Westeros ne peut se comprendre qu'à l'aune des choix géographiques de l'auteur évoqués précédemment. L'austérité et la rudesse du Nord ne sauraient s'accommoder des stratégies en trompe-l'œil des coteries palatiales urbaines. Au-delà du Mur, ce parti pris est encore accentué. Dans ce milieu maudit aux ressources rares, les hommes sont regroupés en clans rivaux. La commensalité restreinte entretient d'incessants conflits pour le contrôle des maigres territoires de chasse. Seul le charisme éphémère d'un roi providentiel, Mance Rayder, peut unir les peuples sauvages pour entreprendre un «Grand Trek» vers les terres plus riches et plus sûres de l'en deçà du Mur, dans le royaume des Sept Couronnes. Westeros semble donc être organisé selon un gradient sud-nord de cohésion spatiale et d'interspatialité des établissements humains rencontrés. Il en résulte un système centre-périphéries assez voisin de celui étudié par Barry CunlifFe pour l'Empire romain et ses voisinages (CunlifFe, 1993).

\section{Les Cités libres d'Essos et l'empire nomade dothraki}

10 À l'est du détroit, les rivages d'Essos sont occupés par des cités libres contrôlées par des oligarchies de marchands. Situées à l'interface entre le littoral de la mer d'Été et le vaste hinterland de la mer Herbeuse, elles concentrent les principaux noyaux de peuplement et d'activités d'Essos. Si leur assise territoriale semble modeste, en revanche, les flottes de navires ou les caravanes élargissent considérablement leurs aires d'influence. Mereen, Yunkaï et Astapor s'inscrivent dans un vaste réseau commercial et leurs rapports État-territoire s'apparentent à l'« État point champ » des cités phéniciennes ou des échelles du Levant. Dans cette économie-monde, proche du système-monde mésopotamien de Guillermo Algaze (1993), l'emprise de ces cités sur 
l'espace alentour combine les logiques de Christaller et de Zipf, à la fois lieux centraux et ville macrocéphale. Les cités de la Baie des Serfs partagent de nombreuses caractéristiques avec les villes mésopotamiennes d'Uruk ou Mari (Legras, 2010) : une population nombreuse et laborieuse qui s'entasse dans un dédale de rues plus ou moins sûres. Le paysage urbain est dominé par une grande pyramide librement inspirée de la Ziggurat d'Ur Nammu d'Uruk. Les membres des oligarchies occupent quant à eux des vastes édifices qui combinent espaces ouverts et grandes salles où s'étalent dans une débauche de luxe les fruits du grand commerce. Cette territorialité réticulaire nourrit à son tour l'intrigue romanesque. L'influence de la «banque de fer» de Braavos ou la guilde des Judicieux attachée à la perte de la princesse Targaryen constituent des facteurs de cohésion spatiale et narrative entre les multiples développements de l'histoire contée par l'auteur. Reste enfin l'empire des Dothraki dont l'influence s'exerce sur la vaste steppe centrale d'Essos. Il s'agit d'un empire nomade constitué de clans unifiés ponctuellement par un « loup bleu » sur le modèle des hordes d'Attila ou Gengis Khan. Pratiquant une économie de razzias ou trouvant à s'embaucher comme mercenaires, ces cavaliers émérites évoluent dans un espace flou aux frontières imprécises. Le seul lieu stable de cet empire est la capitale Vaes Dothraki sise au pied d'une montagne sacrée. Si ce lieu ne saurait être considéré comme une ville au sens commun du terme, il s'apparente toutefois aux sites d'occupation pérenne attestés par l'archéologie chez les peuples nomades d'Asie centrale comme les Sarmates (Lebedynsky, 2007).

Figure 2. Modèle A : les configurations géohistoriques du monde textuel de G.R.R. Martin

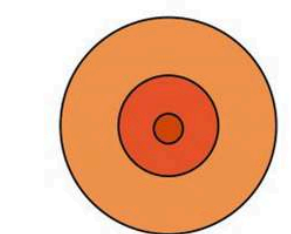

Les auréoles de la cohésion spatiale de Westeros

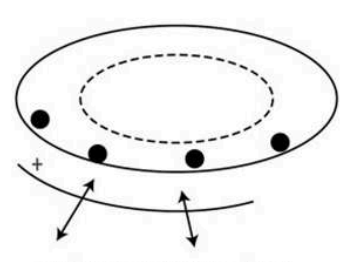

L'archétype «asiatique» d'Essos

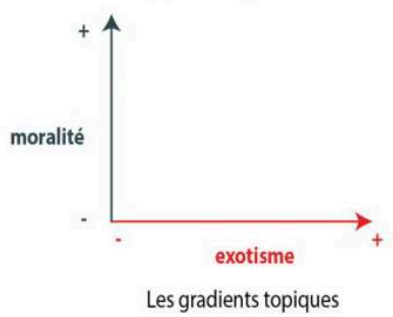

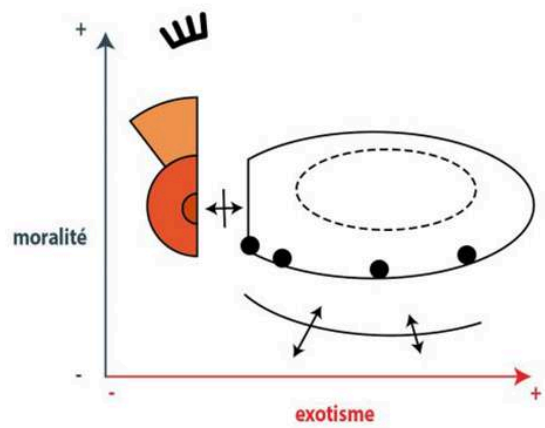

Modèle de l'organisation du monde textuel de Game of Thrones 


\section{Commentaire}

11 Le modèle est construit autour de trois chorèmes élémentaires. Le système centrepériphérie de Westeros est représenté par trois auréoles concentriques qui symbolisent les degrés de la cohésion territoriale de chacun des sous-ensembles régionaux étudiés. Essos est un bloc continental étiré d'où le choix de l'ellipse, les noyaux de peuplement dense sont les points noirs tandis que les pointillés marquent l'aire de nomadisation des Dothraki aux contours incertains. Enfin les gradients topiques d'exotisme et de moralité sont des flèches. L'importance du détroit introduit une dissymétrie dans le modèle sans pour autant provoquer de perturbations majeures.

\section{Pratiques spatiales et trame romanesque}

L'espace de la saga Game of Thrones n'est pas seulement un cadre géographique support d'une intrigue littéraire déterritorialisée. Sa diversité, ses caractéristiques et les pratiques spatiales qui lui sont attachées en font un ressort essentiel au succès de l'histoire. En efFet, ce sont bien les déplacements des différents protagonistes qui alimentent les péripéties du récit, ils sont cependant sous-tendus par des logiques géohistoriques inattendues dans une œuvre d'Heroic Fantasy. Dans cette perspective, le jeu des personnages peut se ramener à trois grands types de pratiques spatiales.

\section{Les déplacements centrifuges à partir de Port Réal}

13 Si le principal centre du pouvoir à Westeros se trouve à Port Réal, celui-ci paraît s'affaiblir. L'incurie des souverains et la faillite des alliances érodent l'influence de Lannister. À terme cet "homme malade » est appelé à se régénérer ou à disparaître. Cette situation constitue une grille de lecture pertinente pour examiner les déplacements d'un certain nombre de personnages qui émaillent le récit de Martin. Tywin Lannister et Jaime Lannister quittent Port Réal pour afFronter l'armée des Nordiens, révoltée après le meurtre d'Eddard Starck, ou pour rafFermir des alliances vacillantes et ramener les bannerets à l'obéissance. Ces déplacements du centre vers la périphérie du royaume sont motivés par une volonté de consolider une domination mise à mal, il s'agit de restaurer la maitrise des distances géographiques et politiques, gage de cohésion spatiale au sein d'une construction politique qui s'apparente à l'« Arkhé » athénien de Périclès ou au bloc soviétique. À l'opposé, les pérégrinations de Tyrion Lannister, le nain, ou du maître des espions Varis en Essos relèvent d'une autre logique. Après avoir tué son père Tywin, Tyrion est contraint de quitter Port Réal pour sauver sa vie. Varis, pour sa part, en fin politique quitte le giron Lannister en déclin pour se rapprocher de Daenerys Thargaryen. L'éloignement, dans ces conditions, ne fait sens que si l'on prend en compte la rupture topographique du détroit qui crée une dissymétrie dans l'aire d'influence des Lannister qui s'exerce surtout sur un espace continental.

\section{Les déplacements centripètes vers Port Réal}

Les déplacements en direction de Port réal constituent l'autre grande pratique spatiale des héros de Game of Thrones. Ils consistent en une série d'expéditions depuis les marges 
septentrionales et orientales vers le cœur de Westeros. Ces déplacements alimentent les épisodes en multiples rebondissements. Après avoir été vendue au Khal dothraki, la princesse héritière de l'ancienne lignée légitime entreprend la lente reconquête du Trône de Fer. Partie des confins de la steppe, elle soumet à son autorité les cités esclavagistes de la côte sud d'Essos et se prépare à envahir Westeros. Au nord, la situation est plus confuse. Le meurtre de Ned Stark, principal féal du défunt souverain Barathéon, ouvre une période de guerre civile féroce entre les principales maisons seigneuriales. Si la comparaison avec la Guerre des Deux Roses qui déchira l'Angleterre entre 1455 et 1485 s'impose naturellement, les renversements d'alliances et l'omniprésence de la vengeance évoquent aussi les nombreuses «faides", ces vendettas médiévales qui épuisèrent nombre de lignages seigneuriaux et préparèrent au renouvellement des élites politiques et à une redéfinition des centres de gravité politique des territoires. La guerre entre les lignages Awans et Waroux en Hesbaye liégeoise entre 1297 et 1335 couta la vie à plus de 30000 hommes et permit à la bourgeoisie, soutien des Awans, de s'affIrmer comme une force politique nouvelle face au Prince Évêque de Liège soutien des Waroux (Masson, 2013). En outre, la trahison primordiale de Joffrey Barathéon-Lannister envers son vassal renvoie à la théorie politique du meurtre du seigneur, nécessaire au rétablissement d'un ordre humain, mais aussi universel, étudiée par Robert Jacob (1990). Autant d'éléments qui, dans l'œuvre de Martin, préparent l'avènement d'un prince caché, Jon Snow, bâtard de Ned Stark et d'une princesse de sang royal. Ses pérégrinations depuis le Mur, espace de relégation géographique et social, vers le château de Winterfeld contiennent une évidente dimension géohistorique. Ce scénario géohistorique est bien connu des spécialistes sous le nom "d'unification par la marge ». De manière inattendue Martin, applique à son monde textuel cette évolution étudiée par Alain Reynaud à propos de la Chine des royaumes combattants (Reynaud, 1992).

\section{Les chevauchées dothraki}

L'étude des pratiques spatiales propres aux Dothraki est inséparable de la prise en compte de leur environnement géographique. Leur domaine de nomadisation est une vaste steppe herbeuse qui ne peut guère fournir de ressources abondantes et régulières à une population sédentaire. Les hordes sont donc contraintes à des tribulations incessantes à la recherche de nouveaux pâturages pour les troupeaux. À côté des déplacements pastoraux, les Dothraki se livrent à de multiples raids dirigés contre des clans rivaux ou vers les cités plus riches de la bordure méridionale de la Mer herbeuse. Toutefois les rivalités endémiques dans le monde dothraki et la disparition précoce du « loup bleu » Khal Drogo ont empêché le déclenchement de grandes invasions de type mongol. Au contraire, les rapports entre les nomades et les sociétés plus denses sont demeurés limités à des phénomènes de turbulences sans impact durable sur la géopolitique régionale avant l'arrivée de la Mère des Dragons (Daenerys Thargaryen). Ces scénarii ont été étudiés par Christian Grataloup dans le chapitre de sa thèse consacré aux interactions des modèles d'invasion au pourtour d'une masse continentale (Grataloup, 1994). 
Figure 3. Modèle B : La diversité des pratiques spatiales dans Game of Thrones, une congruence géohistorique inattendue

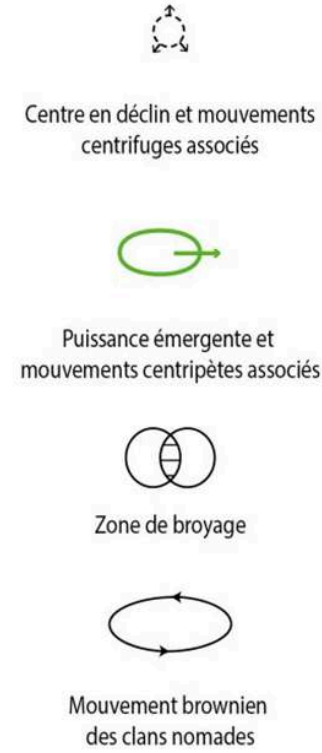

Mouvement brownien
des clans nomades

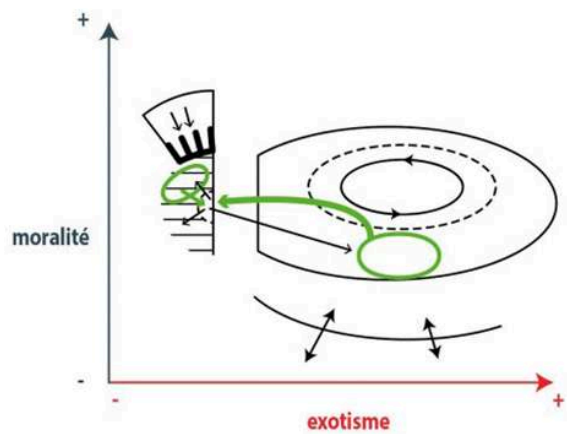

Modèle de la diversité des pratiques spatiales dans Game of Thrones, une congruence géohistorique inattendue

\section{Commentaire}

Modéliser les pratiques spatiales des protagonistes de Game of Thrones revient à identifier un certain nombre de logiques géopolitiques représentées par les 5 chorèmes qui sont à la base du modèle de synthèse. L'afFaiblissement du centre est symbolisé par le cercle en pointillé tandis que l'émergence d'une nouvelle aire de puissance qui transcende les établissements humains antérieurs est soulignée par un cercle plus épais. La faible cohésion politique de l'aire dothraki est également figurée par des pointillés, car elle ne pèse guère au-delà du cœur d'Essos. La taille des flèches montre à la fois l'importance et l'incidence des déplacements étudiés.

Cette contribution espère avoir ouvert un champ de questionnement légitime sur la place de la géographie comme élément à part entière de la construction narrative dans une œuvre de fiction comme Game of Thrones. En efFet, les partis pris géographiques de l'auteur nourrissent tant la trame romanesque que la psychologie des personnages et contribuent à donner une véritable épaisseur spatiale dramatique à l'œuvre. Les éléments de géographie mobilisés par Martin relèvent de différents paradigmes, classiques ou plus récents, qui témoignent de l'imprégnation d'une culture géographique diffuse de l'auteur. $\mathrm{Si}$, de prime abord, le sujet de cet article peut apparaître surprenant, il témoigne, au contraire, de l'importance de la géographie jusque dans des champs culturels inattendus. 


\section{BIBLIOGRAPHIE}

ALGAZE G. (1993). The Uruk World System, the Dynamics of Expansion of early Mésopotamian Civilization. Chicago: University of Chicago Press, 162 p. ISBN 9780226013817

BRUNET R., FERRAS R., THÉRY H. (1993). Les mots de la géographie. Montpellier : Reclus, Paris : La Documentation Française. ISBN 9782110059437

CUNLIFFE B. (1993). La Gaule et ses voisins. Paris : Picard, 256 p.

Géographie et littérature. http://cafe-geo.net/geographie-et-litterature-2/

GRATALOUP C. (1994). L'espace de la transition. Essai de géohistoire chorématique. Thèse de doctorat, vol. 2, Université de Paris I.

JAСоB R. (1990). « Le meurtre du seigneur dans la société féodale, la mémoire, le rite et la fonction ». Annales ESC, vol. 45, n² 2, p. 247-263.

LEBEDYNSKY I. (2007). Les nomades : les peuples nomades de la steppe des origines aux invasions mongoles (IX siècle av. J.-C.-XIII siècle ap. J.-C.). Paris : Éditions Errance, coll. "Civilisations et culture ", 256 p. ISBN 978-2-87772-346-6

LEGRAS B. (2010). « 20 ans de réflexions sur la ville antique, tendances historiographiques actuelles ». Histoire urbaine, $\mathrm{n}^{\circ}$ 29, 3/2010, p. 1 29-131.

MASSON C. (2013). « La guerre des Awans et des Waroux. Une vendetta en Hesbaye liégeoise (1297-1335) ». Le Moyen Age. Revue d'histoire et de philologie, t. CXIX, n² 2, p. 403-448.

REYNAUD A. (1992). Une géohistoire, la Chine des Printemps et des Automnes. Montpellier : Reclus, 220 p. ROBIC M.-C., ROSEMBERG M. (dir.). Géographier aujourd'hui. Enseigner la géographie au collège et au lycée. ADAPT/SNES, $338 \mathrm{p}$.

ROLET S. (2014). Le trône de fer. Le pouvoir dans le sang. Tours : Presses universitaires François Rabelais, 350 p. ISBN 9782869063792

\section{AUTEUR}

\section{PHILIPPE MOYEN}

Professeur agrégé Histoire-Géographie, lycée Sévigné, Charleville-Mézières 